Trascender, Contabilidad y Gestión. Vol. 5, Núm. 14 (mayo - agosto del 2020). Universidad de Sonora. Departamento de Contabilidad. ISSN: 2448-6388. Reserva de Derechos 04-2015-04172070800-203.

\title{
Tecnologías para la mejora de la transparencia
}

\author{
Technologies for improving transparency
}

Marco Antonio Ross Ibarra ${ }^{1}$

Recibido: 15 de diciembre de 2019.

Aceptado: 20 de marzo de 2020.

DOI: https://doi.org/10.36791/tcg.v0i14.84

JEL: M15. Gestión de la tecnología de la información.

\section{Resumen}

En este trabajo se analiza la transparencia, así como se observan las herramientas de las tecnologías de la información que han estado mejorando la eficiencia de la transparencia en múltiples campos.

Debemos aclarar, que la transparencia no es algo nuevo, lo que, si es novedoso, es como se ha adaptado dentro de múltiples campos profesionales, usualmente pensamos en la transparencia como algo único del gobierno y su rendición de cuentas, pero la transparencia es algo mucho más adaptable.

A través de este trabajo se mostrarán múltiples ejemplos de las tecnologías de la información que convierten a la transparencia en una herramienta para la mejora continua, para ofrecer sugerencias dentro de los múltiples ámbitos profesionales, demostrando los beneficios que han traído en otras áreas.

\section{Abstract}

This paper analyzes transparency, and it looks the tools that technology of information that are making great advances in the efficiency and transparency in multiple fields.

We should clarify that transparency is not something new, what is indeed new, is how it has been adapted into multiple professional fields, usually we think in transparency as a tool of government, but it is something much bigger.

Through this work we get to see multiple examples of technologies that turn transparency into a tool for the continue advancement to offer advice through multiple fields, showing the benefits that it brings to other areas.

Keywords: transparency, technology, information.

Palabras clave: transparencia, tecnologías, información.

\footnotetext{
${ }^{1}$ Marco Antonio Ross Ibarra. Contador Público. Master en Fiscalización y Control Gubernamental. Auditor del Instituto Superior de Auditoría y Fiscalización. Correo: marcorossmx@gmail.com 


\section{Antecedentes}

Las tecnologías de la información no son un tema nuevo, sabemos que están presentes en múltiples campos y en muchas áreas, lo que no sabemos es cómo funcionan estas en otros campos, o incluso como se han tornado en herramientas para la transparencia.

Estas tecnologías vienen de maneras tan sencillas como la entrada de los softwares estadísticos que nos permiten la captura de datos para el desarrollo de bases de datos que permiten análisis históricos del desarrollo de los proyectos o estudios que se manejan.

La transparencia es una herramienta que se necesita y beneficia a todos los que se involucran, y a veces no se logra notar, pero está ahí. Un caso interesante fue el del 2000 en el estudio publicado "To Err is Human: Building a Safer Healthcare System (Institute of Medicine, 2000)".

El estudio publicado en ese entonces, hablaba de errores médicos que costaron vidas y pudieron haberse solucionado de manera sencilla, simplemente con mejor comunicación entre paciente y doctor.

La National Patient Safety Foundation, (NPSF por sus siglas en Ingles) escribió en 2015 un reporte donde hablaba de la transparencia, ofreciendo la definición que se ha mantenido a la fecha por los médicos: Transparency the free, uninhibited Flow of information that is open to the scrutiny of the others (National Patient Safety Foundation, 2015, p. vii).

Y es esta definición que yo aprovecho, puesto que toca un punto muy importante, la transparencia es el flujo libre desinhibido para la información, y en nuestro mundo tenemos que aprovechar las mismas tecnologías de la información para esto.

Es por eso que la transparencia es algo que no solo se enfoca en un área, si no que en múltiples áreas permite una labor más sencilla, un ejemplo de esto es como se aplican los recientes smart contracts. Estos son contratos digitales transparentes. La información en ellas es clara, y aunque cualquiera puede verlos, están altamente encriptados para protegerlos de la edición de un tercero.

Don Tapscott autor del libro Wikinomics, y el libro más reciente en 2016 Blockchain Revolution: How the Technology Behind Bitcoin is Changing Money, Business and the World. Toca en su libro sobre la blockchain, un asunto muy particular, que es la diferencia entre lo que es la privacidad y la libertad.

"It is no accident that Germany is the leading privacy and data protection country in the world. They had to endure the abuses of the Third Reich and the complete cessation of all of their freedoms, which started with the complete removal of their privacy. When that ended, they said, 'Never again.'” (p. 27) -Ann Cavoukian, Executive Director of the Privacy and Big Data Institute at Ryerson University (Tapscott y Williams, 2016).

La privacidad y la transparencia no chocan realmente entre ellas. La transparencia en cambio se define como la cualidad de transparente, concepto que hemos tomado para decir que la transparencia es una cualidad de que las cosas se muestran tal y como son sin ocultar nada.

Estos conceptos podrían tomarse como contradictorios entre ellos, pues pedimos transparencia, y algunos suponen que esa transparencia afectara la vida privada. La verdad es que no funciona así, la transparencia implica sepamos como suceden las operaciones y en qué consisten, pero sin afectar la privacidad de los miembros de la operación.

La transparencia supone como se mueven los números, que sepamos como funciona todo dentro del sistema y que se nos pueda explicar de manera comprensible para todos. Esto miso no toca la privacidad, no afecta a los involucrados en las operaciones dentro del sistema. 
Sabemos sus roles y sus obligaciones, la transparencia nos revela esa información, pero en ningún momento expone la vida privada de las mismas, solo lo que realmente nos concierne saber por su puesto y obligación dentro del sistema del que forman parte.

La privacidad y la transparencia no son en ningún momento enemigas, la transparencia respeta la privacidad de las personas puesto que su foco de aplicación no recae en estas, si no en el sistema del que se forme parte como anteriormente se menciona.

Citando una vez más a Don Tapscott, en su libro Wikinomics: How mass collaboration changes everything (Tapscott y Williams, 2010). Nos muestra como en nuestro mundo altamente globalizado las cosas han cambiado, y necesitamos más transparencia para el control de las mismas operaciones, así como aprovechar las ventajas tecnológicas presentadas.

La transparencia en ningún punto contradice la privacidad de las personas, y con las tecnologías y las herramientas nuevas podemos manejar de manera correcta estas para poder sacar el máximo provecho a la transparencia.

Las tecnologías de la información han cambiado como manejamos las operaciones, han realmente traído un panorama nuevo a lo que estábamos acostumbrados.

Hemos visto también el rol que nos da la transparencia en los grandes negocios (Nunkoo, Ribeiro, Sunnassee y Gursoy, 2018), así como hemos visto en ejemplos históricos como la transparencia abre múltiples puertas (Smith, 2018).

Incluso en el mundo digital la transparencia y la privacidad toman roles importantes que se respetan y se ven en las políticas mismas de los países (Crain, 2016).
Oliveira y Cappelli ya han observado el tema de las organizaciones y su transparencia, dándonos los mismos ejemplos de las ventajas anteriormente analizadas por otros (De Oliveira Almeida, Cappelli y Maciel, 2018).

Incluso la transparencia tiene un efecto de mediación para las relaciones sociales y corporativas de los negocios (Baraibar-Diez y Luna, 2018), hemos visto un mundo en el que la tecnología nos abre puertas, y la transparencia las mantiene abiertas, y lo que es más, las expande.

Actualmente incluso vemos ejemplos de la transparencia como herramienta de confianza con los consumidores en el mercado en línea que fluctúa a altas velocidades (Stevens et al., 2018). Un mercado que cabe mencionar, se ha tomado tan enserio la transparencia que cada vez se vuelve más claro para los consumidores.

Podemos ver esto en hechos tan sencillos como que ahora cuando se compra algo en línea se puede rastrear, se puede saber en todo momento a través de que aduanas ha pasado el paquete, se puede saber quién lo recibe y a qué hora. La transparencia nos da confianza en el servicio, al darnos una visión clara de los procesos.

La transparencia es una herramienta tan grande como la tecnología. Los miedos de las personas a comprar en línea antes eran más grandes, y bastante justificables.

Ahora tenemos seguridad y confianza, y esta no nace solamente de la calidad del servicio, se basa en el hecho tangible de que se puede saber en todo momento lo que pasa en el proceso. Es esto lo que hace que la tecnología de la blockchain sea tan fuerte.

La confianza que viene con la transparencia, la blockchain son los datos de la información que todos pueden ver, en todo momento, sin restricciones, y es lo mismo que hace tan útiles a los smart contracts. 
Un smart contract tiene la función de que se puede observar igual en todo momento y se puede rastrear su progreso. Pero, lo que es más, elimina el concepto de la intermediación de partes. A veces las corporaciones tienen todo el acceso a la información, lo cual dificulta la transparencia.

Con estas tecnologías se elimina la necesidad de intermediarios para el acceso a la información, puesto que la información es accesible en todo momento gracias a los avances de las tecnologías de la información y las políticas de transparencia que le dan confianza y fuerza en el respaldo a su uso. Lo cual me lleva al problema de esta investigación.

\section{El problema de investigación}

Sabemos que en ocasiones los problemas de la transparencia son más relacionados a cuestiones actitudinales y de conocimiento (Král y Cuskelly, 2018), $\mathrm{y}$ en lo referente a los problemas de las nuevas tecnologías recae en el hecho de que no hay el personal capacitado para su implementación.

Cuestiones como esta traen consigo un atraso al paso del avance tecnológico y de la aplicación de nuevas tecnologías para la facilidad del desarrollo. Hay tecnologías muy nuevas, pero se tiende a desconfiar, o no se gusta de poner a prueba.

La brecha tecnológica también juega un papel importante en las dificultades para la aplicación de las nuevas Tecnologías de Información y Comunicación (TIC) sabemos que hay múltiples beneficios de buscar el avance tecnológico, pero hay falta de infraestructura necesaria, en el 2015, se inició el proyecto de red de México Conectado, que si bien ha ido en avance aún no se cumple el objetivo de red para todos.
Las tecnologías ya están existentes, y su aplicación es posible, pero se debe tener un equipo para ellas, lo cual me lleva a la justificación de este trabajo.

\section{Justificación}

Hay múltiples áreas donde se puede aprovechar las nuevas tecnologías para apoyar a la transparencia, hay muchos campos de aprovechamiento. Tenemos antecedentes muy recientes de las ventajas de la transparencia, y es una herramienta que no es difícil de aplicar, no es realmente intrusivo en las labores, incluso permite mejores avances. Y combinándola con las herramientas de las TIC (Tecnologías de la Información y la Comunicación), nos permite una mejora constante de las mismas.

Además, las tecnologías para la transparencia no chocan con la privacidad de los negocios ni de las personas involucradas, la transparencia es una herramienta que permite generar no solo beneficios, si no también permite generar una mayor confianza con las personas. Y las tecnologías de la transparencia se enfocan en mantener esa confianza y hacerla crecer al demostrar los beneficios.

Sabemos que hay ventajas en los negocios transparentes (Merlo, 2018), ventajas que no se están aprovechando completamente, es por eso mismo que las tecnologías son un paso necesario, para poder sacar al máximo la transparencia y las ventajas que hay, y para eso se requiere un avance en las mismas.

El objetivo es dar a conocer las herramientas de las nuevas TIC que permiten mejores avances en la búsqueda de una mayor transparencia.

Dar a conocer la información de los smart contracts y la blockchain que permitirían avances en campos de múltiples ámbitos en nuestro país. 
Dar a conocer, que podemos aplicarlas tecnologías a plataformas que ya tenemos funcionando en el país, y que podrían mejorar a través del uso de estas tecnologías.

De tal manera, que un estudio cualitativo permite observar los beneficios que se han obtenido, o los cambios traídos por la aplicación de la transparencia en los negocios, dejando en claro y evidenciado cómo funciona en los negocios o distintos sectores, en donde se aplican las nuevas herramientas de las TIC, así como los campos de oportunidad de las nuevas tecnologías.

\section{Conclusiones}

Los smart contracts son herramientas que pueden accederse fácilmente, e igual el sistema de blockchain, lo que falta para hacerlos funcionar son la TIC, y su desarrollo actual, de alguna manera todo se resume en fondos e infraestructura.

Hay grandes campos de oportunidad para estas herramientas que nos permitirían trabajar con mayor transparencia y aumentar la confianza que se puede generar. Solo se necesita, la inversión inicial de esas tecnologías.

Tecnologías que incluso los mismos bancos pueden aprovechar, la blockchain es una herramienta muy fuerte en la materia de la transparencia que permite se vean las

operaciones, y lo que es más, que no están en control de un solo ente. Lo que permite que se maneje de una manera más sencilla para todos.

Las herramientas están disponibles. Solo falta poder aplicarlas apropiadamente para traer más transparencia a través de ellas.

Hay un campo de posibilidad muy grande abierto en México, tenemos plataformas digitales que pueden beneficiarse altamente de la aplicación de las tecnologías existentes, adaptables a nuestro sistema.

\section{Referencias}

Baraibar-Diez, E., y Luna, L. (2018). The mediating effect of transparency in the relationship between corporate social responsibility and corporate reputation. Revista Brasileira de Gestao de Negocios, 5-21.

Crain, M. (2016). The limits of Transparency: Data Brokers and commodification. Sage Journal.

De Oliveira Almeida, G., Cappelli, C., y Maciel, C. (2018). Organizational Transparency en Encyclopedia of Information Science and Technology. Brazil. https://doi.org/10.4018/978-1-5225-2255-3.ch065

Institute of Medicine. (2000). To Err is Human. Washington: National Academy Press.

Král, P., y Cuskelly, G. (2018). A model of Transparency: Determinants and implications of transparency for national sport organizations. European Sport Management Quarterly, 18(2). Pp. 237-262.

Merlo, O. (2018). The benefits and implementation of performance transparency: The why and how of letting your customers 'see through' your business. Business Horizons, pp 73-84.

National Patient Safety Foundation. (2015). Shining a Light: Safer health care through transparency. Boston: NPSF.

Nunkoo, R., Ribeiro, M. A., Sunnassee, V., y Gursoy, D. (2018). Public trust in mega event planning institutions: The role of knowledge, transparency and corruption. Tourism Management, pp 155-166. 
Smith, A. (2018). Prospect for a transparency revolution in the field of business history.

https://www.tandfonline.com/action/showCopyRight?scr oll=top\&doi=10.1080\%2F000767 91.2018.1439019

Stevens, J. L., Spaid, B. I., Breazeale, M., y Esmark Jones, C. L. (2018). Timeliness, transparency, and trust: A framework for managing online customer complaints. Business Horizons, 375-384.
Tapscott, D., y Williams, A. D. (2010). Wikinomics: How Mass Collaboration Changes Everything. Portfolio. Don Tapscott, A. T. (2016). Blockchain Revolution: How the Technology Behind Bitcoin is Changing. Money, Business and the World. 